\title{
CdS Nanorods Imbedded in Liquid Crystal Cells for Smart Optoelectronic Devices
}

\author{
Kuan-Ju Wu, Kung-Ching Chu, Chih-Yu Chao, and Yang-Fang Chen* \\ Department of Physics, National Taiwan University, Taipei 10617, Taiwan \\ Chih-Wei Lai, Chia-Cheng Kang, Chun-Yen Chen, and Pi-Tai Chou \\ Department of Chemistry, National Taiwan University, Taipei 10617, Taiwan
}

Received March 7, 2007; Revised Manuscript Received May 25, 2007

\begin{abstract}
A novel nanocomposite device, which consists of liquid crystals and semiconductor nanorods, has been designed and fabricated. It reveals a very unique and useful behavior in that the polarization of the emission from semiconductor nanorods can be controlled by an external bias. The large magnitude of polarization anisotropy of 0.63 can be quantitatively interpreted very well in terms of the dielectric contrast between semiconductor and liquid crystal. Our approach is quite general, which is applicable to other nanomaterials, and it utilizes the currently mature liquid crystal display technology. The results open up new possible applications for one-dimensional semiconductor nanostructures in smart optoelectronic applications, including optical switches, integrated photonic devices, as well as electrochromatic gadgets in the near future.
\end{abstract}

One-dimensional semiconductor nanostructures are intriguing materials both for academic interest and industrial application. It has been shown that semiconductor nanowires own unique thermal, electric, optical, and mechanical properties, which have great potential applications, including novel solar cell architectures, nanolasers, computational technology, communication, and biological science and technology. ${ }^{1-5}$ To achieve their full potential for future practical applications, the next step is to incorporate the semiconductor nanowires with existing technologies and to integrate them into unique electric and optoelectronic devices. Herein, we report a novel composite device, which consists of liquid crystals (LCs) and semiconductor nanorods. This newly designed device has a unique feature in that the polarization of the emission from semiconductor nanorods can be finetuned by an external bias. It is well-known that the ability to control the polarization of the emission from an optoelectronic device is very important because it offers an excellent opportunity to manipulate the communication of information and images. Moreover, in this device, semiconductor nanorods can form a well-aligned array with the assistance of liquid crystals, ${ }^{6}$ which is otherwise difficult to achieve by using other methods so far. Notably, this novel device utilizes the currently mature liquid crystal display (LCD) technology, ${ }^{7}$ which is quite feasible for practical

* Corresponding author. E-mail: yfchen@phys.ntu.edu.tw. Telephone: 886-2-33665125. Fax: 886-2-23639984. Address No. 1, Sec. 4 Roosevelt Road, Department of Physics, National Taiwan University, Taipei 106, Taiwan, Republic of China. applications. Our strategy is very general and universal and is readily applied to many other nanomaterials. It therefore opens new possibilities for semiconductor nanowires in smart optoelectronic applications including electrochromatic gadgets, optical switches, integrated photonic devices, etc.

To illustrate the design strategy, CdS nanorods were chosen as a prototype. CdS rods were prepared according to the method reported by Alivisatos and co-workers ${ }^{8}$ with slight modification. Briefly, a sulfur stock solution containing $32 \mathrm{mg}$ of sulfur ( $1 \mathrm{mmol}$ ) was prepared in a glovebox by dissolving sulfur powder in $2 \mathrm{~mL}$ of tri- $n$-octylphosphine (TOP). The sulfur stock solution was then brought out of the glovebox in a vial sealed with a rubber subseal. A mixture of $\mathrm{CdO}$ (300 mg, $2.34 \mathrm{mmol}$ ), octadecylphosphonic acid (ODPA) (910 mg, $2.72 \mathrm{mmol}$ ), and octylphosphonic acid (OPA) (350 mg, $1.8 \mathrm{mmol}$ ) were heated in a three-neck flask to $290{ }^{\circ} \mathrm{C}$ under Ar flow. When the solute was completely dissolved, the stock solution of tri- $n$-octylphosphine sulfur (TOPS) mixtures was quickly injected into the hot solution. $\mathrm{CdS}$ rods were formed and appeared to be in yellow-brown solution after reacting for $3 \mathrm{~min}$. The prepared $\mathrm{CdS}$ rods were further purified by centrifugation and double reprecipitation from isopropanol. Figure 1shows transmission electron microscopy (TEM) images of CdS nanorods. The length and the diameter of the $\mathrm{CdS}$ rods are 40 and $5 \mathrm{~nm}$ on average, respectively.

LC cells were fabricated according to a standard procedure, ${ }^{7}$ which consists of two counter indium tin oxide (ITO)- 


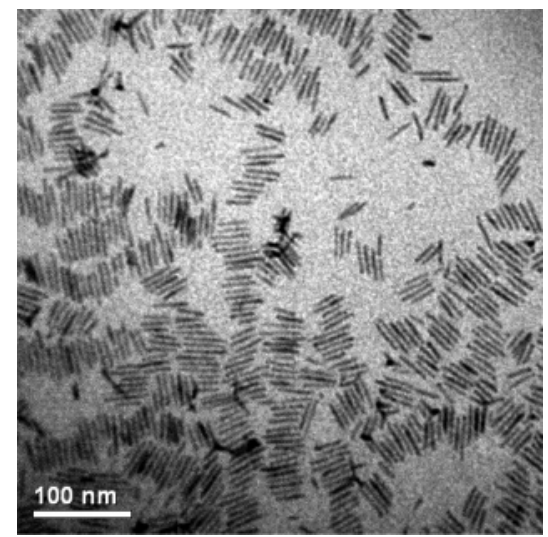

Figure 1. Transmission electron microscopy image (TEM) of CdS nanorods.

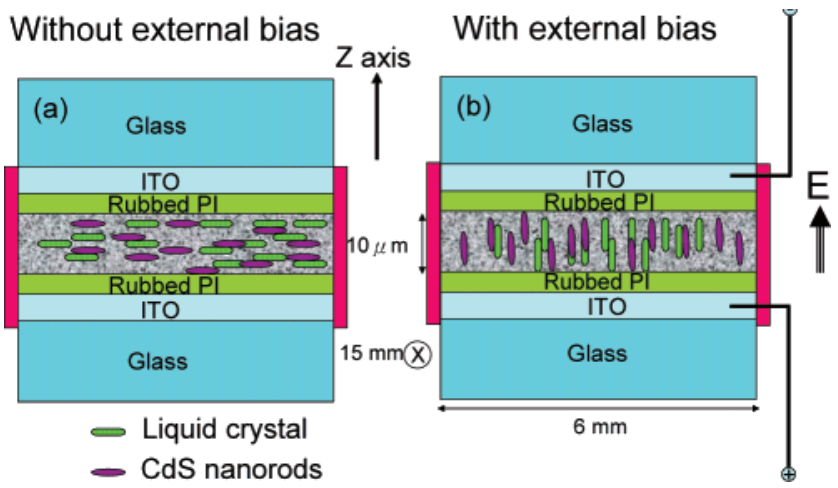

Figure 2. Structure of the fabricated device consisting of liquid crystals and $\mathrm{CdS}$ nanorods with and without an applied electric field. Note that the applied electric field is normal to the cell plane.

coated glass substrates with a rubbed polyimide (PI) alignment layer. First, we dropped the suspension solution (solvent: toluene) of CdS nanorods onto the rubbed PI layer until CdS nanorods were dried. After the combination of top and bottom treated substrates in the same rubbed direction, the nematic LC E7 from Merck was injected into the assembled substrates. A supersonic treatment was employed to obtain a better mixture of the LC molecules and $\mathrm{CdS}$ nanorods. Figure 2 shows a schematic of the side-view structure of the LC cell with and without external electric field. The dimension of the LC cell is $6 \mathrm{~mm} \times 15 \mathrm{~mm} \times$ $10 \mu \mathrm{m}$.

An alternating electric field at $1 \mathrm{kHz}$ frequency is applied across the sample ( $z$-axis) to avoid the induced separation of charged impurities. A schematic diagram of the experiment setup for the optical measurement is shown in Figure 3. A laser beam with $374 \mathrm{~nm}$ is focused into a microscope and used to excite CdS nanorods from the top side of the LC cell. The luminescence from CdS nanorods is again focused into the microscope and then passes through an analyzer, a depolarizer, and enters a TRIAX 320 spectrometer. Finally, a high-response $\mathrm{H} 5783$ photomultiplier tube is employed as the detector. The analyzer in front of the entrance slit of the spectrometer is used to reveal the polarization of the emission from CdS nanorods, and the depolarizer is for eliminating any possible systematic error in the polarization measurement

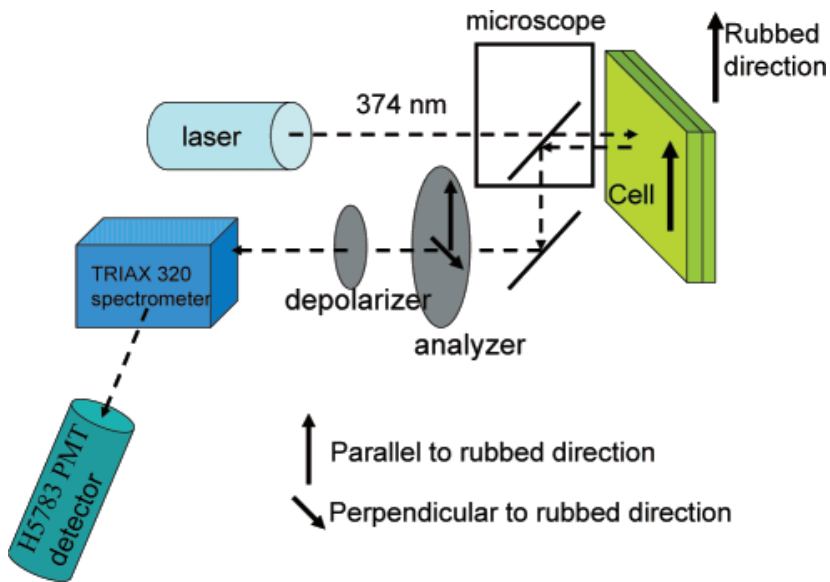

Figure 3. Schematic diagram of the experimental setup used in the optical measurement.

arising from experimental apparatuses. All experiments were performed at room temperature.

For the purpose of comparison, we have also fabricated LC cells containing CdSe nanoparticles instead of $\mathrm{CdS}$ nanorods. With a slight modification of the previous procedure, preparation of the $\mathrm{CdSe}$ core from $\mathrm{CdO}$ was done. ${ }^{9}$ Briefly, a Se injection solution including $0.079 \mathrm{~g}$ of Se (1 mmol) was prepared by dissolving Se powder in $0.3 \mathrm{~mL}$ of tri- $n$-butylphosphine (TOP)) and diluted with $1.681 \mathrm{~g} \mathrm{di-}$ $n$-octylamine (HDA). A mixture of $\mathrm{CdO}(0.0128 \mathrm{~g}$, $0.10 \mathrm{mmol})$ and stearic acid $(0.114 \mathrm{~g}, 0.40 \mathrm{mmol})$ was gently heated in a three-neck flask to $140{ }^{\circ} \mathrm{C}$ under $\mathrm{Ar}$ flow. When the mixture was completely dissolved and clear, it was allowed to cool to room temperature. Finally, $1.94 \mathrm{~g}$ of the TOPO and HDA was put into the flask, and the mixture was heated to $320{ }^{\circ} \mathrm{C}$. The Se injection solution was quickly injected into the hot reaction solution. The reaction mixture was allowed to cool to $290{ }^{\circ} \mathrm{C}$ for the growth of the CdSe nanocrystals. Sizes of CdSe QDs $(3.5 \mathrm{~nm})$ were obtained by the time period (about $60 \mathrm{~s}$ ) required to end the reaction by cooling. CdSe QDs were then precipitated out from the growth solution by adding methanol.

In Figure 4, the micro-PL spectra reveal a giant anisotropic behavior with an intensity ratio of about 5.0 for the analyzer parallel (dashed line) and perpendicular (solid line) to the rubbed PI direction without external bias. The inset is the variation of overall micro-PL intensity as a function of analyzer angle, which exhibits a periodic $\cos ^{2} \theta$ dependence on angle as one should expect. It clearly shows that the intensity of the micro-PL spectra of the parallel one is much larger than that of perpendicular one. This result implies that $\mathrm{CdS}$ nanorods are directed along the orientation of LC molecules, which is parallel to the rubbed PI direction. The alignment of CdS nanorods is caused by the result of LC infiltration. Recent studies indicate that the orientation dependent force is caused by anisotropic property of aspherical particles. ${ }^{10-16}$ There are usually two types of anchoring behavior at the LC interface. Homeotropic anchoring describes the director of LCs perpendicular to the surface and planar anchoring parallel to the surface. ${ }^{7}$ Inorganic surfaces are known to prefer planar anchoring. ${ }^{14,15}$ The 


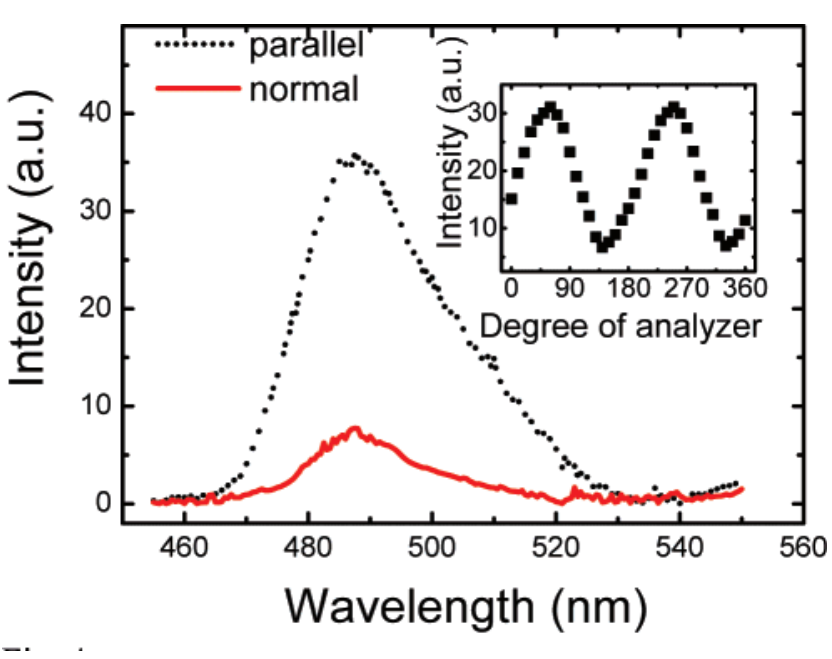

Figure 4. Microphotoluminescence spectra with the analyzer parallel (dashed line) and perpendicular (solid line) to the rubbed PI direction without an external electric field of CdS nanorods and LCs composite. The inset is the variation of overall microphotoluminescence intensity as a function of the angle of analyzer.

fundamental interaction between CdS nanorods and LCs is anchoring that makes LCs attach on CdS nanorods. The tangential boundary condition and the continuum property of LCs is expected to form a north-south pole-type morphology, and the north-south pole should be two sides of the CdS nanorods. ${ }^{17,18}$ Hence, the orientation of $\mathrm{CdS}$ nanorods must be the same with that of LCs on the surface of CdS nanorods. It is well-known that the substrate with a thin PI coating will align LCs along the rubbed direction. ${ }^{7}$ According to elastic continuum theory, all LCs must align in the same direction. ${ }^{7}$ Consequently, the $\mathrm{CdS}$ nanorods will also align along the rubbed PI direction due to the anchoring force between LCs and nanorods. On the basis of the simple model proposed by Burylov and Raikher, ${ }^{19}$ a rough estimate of the alignment energy of one-dimensional nanostructures in LCs can be very large. For example, the alignment energy for the mixture of carbon nanotubes and LCs is given by $3.8 k T$, where $k$ is the Boltzmann's constant and $T$ is temperature ${ }^{6}$ For the case of our experimental condition at room temperature, the alignment energy can reach up to $100 \mathrm{meV}$, which is quite a large energy. It has been pointed out that the alignment energy is underestimated and it does not involve specific intermolecular interaction. ${ }^{19}$ Therefore, the well alignment of $\mathrm{CdS}$ nanorods along the orientation of LC molecules seems quite plausible as shown in our result.

To further confirm that the obtained anisotropy is indeed a result of the well-aligned CdS nanorods instead of the anisotropic property of LC molecules, we have replaced the one-dimensional CdS nanorods in the LC device by the zerodimensional CdSe nanoparticles. For this composite device containing LC and CdSe nanoparticles, the polarization of the emission arising from CdSe nanoparticles is negligibly small, as shown in Figure 5. Here, the diameter of the nanoparticles is about $3.5 \mathrm{~nm}$, and the concentration is about $1.0 \times 10^{11} \mathrm{~cm}^{-3}$. We thus may conclude that the polarization of the emission from the LC cell containing CdS nanorods is due to the nature of the geometric anisotropy of a rod,

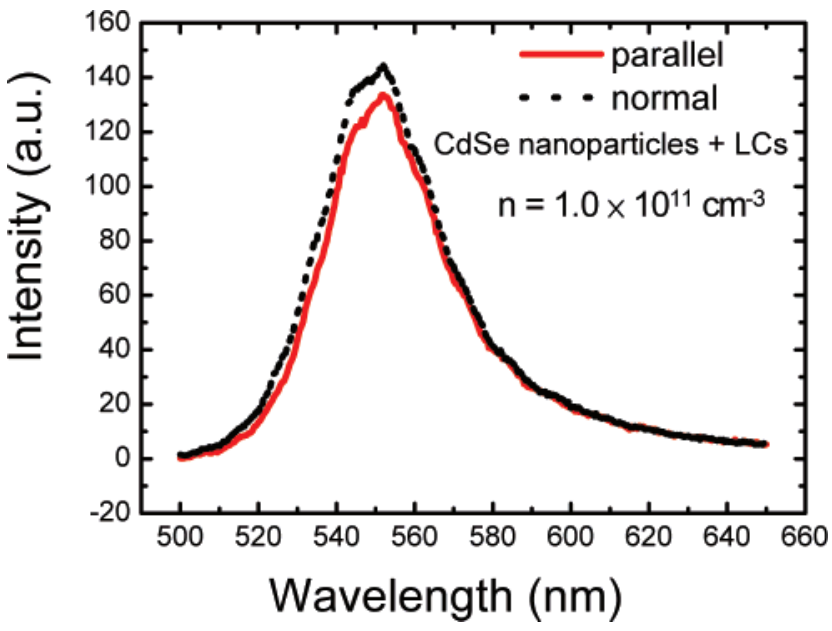

Figure 5. Microphotoluminescence spectra with the analyzer parallel (dashed line) and perpendicular (solid line) to the rubbed PI direction without an external electric field of CdSe nanoparticles and LCs composite.

while the absence of the polarization in the emission of CdSe nanoparticle is because of its spherical symmetry. Thus, we can exclude the origin of the optical anisotropy is caused by the birefringence of the LC. In addition, this result also rules out the possibility that the observed anisotropy is due to the systematic error of our measurement equipments.

The degree of polarization can be calculated by $\rho=$ $\left(I_{\|}-I_{\perp}\right) /\left(I_{\|}+I_{\perp}\right)$, where $I_{\|}$and $I_{\perp}$ represent the polarization parallel and perpendicular to the rubbed PI direction, respectively. The resulting polarization ratio of 0.63 is quite large compared with that of the reported value of less than 0.1 for the nanowire samples. ${ }^{20-21}$ Optical anisotropy has been attributed to the effect of quantum confinement in nanowires. ${ }^{20}$ However, it yields a value smaller than our observation. As for a rationalization, the polarization obtained here can be explained in terms of the dielectric contrast between the semiconductor nanorods and LC molecules. ${ }^{22}$ Because the size of CdS nanorods is much smaller than the emission wavelength, the dielectric field $(E)$ inside the CdS nanorods can be treated as a plane wave with an amplitude modulated on the scale of the nanorods diameter due to the usual boundary conditions. ${ }^{22-25}$ When the polarization of the emission light $\left(E^{\mathrm{in}}\right)$ is parallel to the rods, the modulation of the electric field is negligible compared with that of the $E^{\text {in }}$ perpendicular to the rods due to a large aspect ratio of about 8. Accordingly, the amplitude of the electric field inside the rods has the form:

$$
E_{\|}=E_{\|}^{\mathrm{in}}, E_{\perp}=\delta E_{\perp}^{\mathrm{in}}, \quad \delta=\frac{2 \epsilon_{\mathrm{s}}}{\epsilon+\epsilon_{\mathrm{s}}}
$$

where $\epsilon$ and $\epsilon_{\mathrm{s}}$ are the dielectric constants of the nanorods and LCs, respectively. ${ }^{25}$

The degree of linear polarization $\rho$ is thus expressed as

$$
\rho=\frac{I_{\|}-I_{\perp}}{I_{\|}+I_{\perp}}=\frac{\left|E_{\|}\right|^{2}-\left|E_{\perp}\right|^{2}}{\left|E_{\|}\right|^{2}+\left|E_{\perp}\right|^{2}}=\frac{1-\delta^{2}}{1+\delta^{2}}
$$



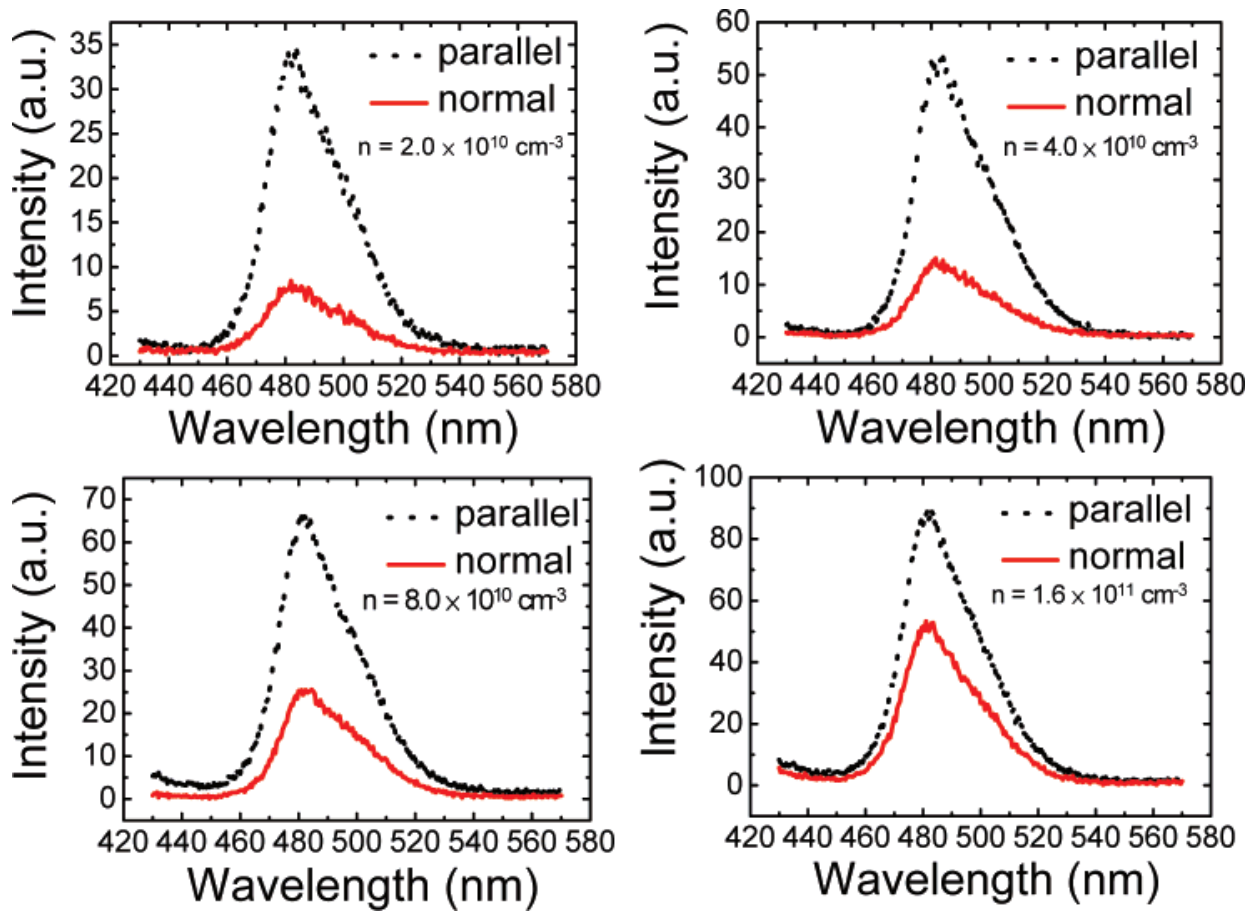

Figure 6. Microphotoluminescence spectra with the analyzer parallel (dashed line) and perpendicular (solid line) to the rubbed PI direction without an external electric field of LCs and CdS nanorods composite which have one, two, four, and eight CdS drops.

Applying the dielectric constant of CdS (bulk $=5.75)$ and LC $\left(\epsilon_{\|}=1.74, \epsilon_{\perp}=1.52\right)$, the theoretical degree of polarization is deduced to be 0.65 , which is in agreement with our experimental result of 0.63 .

It is interesting to see to what extent the addition of CdS nanorods does not disturb the well alignment of LC molecules and reduce the optical anisotropy of CdS nanorods. To resolve this issue, we have performed an additional experiment with different concentrations of $\mathrm{CdS}$ nanorods. As shown in Figure 6, it is found that the polarization initially remains constant and then decreases with increasing concentration of CdS nanorods, with the value of polarization ratio of $63 \%$ in $2 \times 10^{10} \mathrm{~cm}^{-3}$ and $25 \%$ in $1.2 \times 10^{10} \mathrm{~cm}^{-3}$. The concentration of the CdS nanorods was estimated by the corresponding TEM images as shown in Figure 1. Because the concentration of LC molecules is about $10^{21}$ $\mathrm{cm}^{-3}$, we therefore estimate that, in order to have the maximum optical anisotropy, the optimized concentration of $\mathrm{CdS}$ nanorods in the E7 LC cell is about one nanorod per $10^{10} \mathrm{LC}$ molecules. The decrease of the polarization ratio in higher concentration of $\mathrm{CdS}$ nanorods may be attributed to the fact that the driving force to align the nanorod is not enough or the nanorods can now disturb the alignment of LCs along the rubbed PI direction. It is worth noting that, under our experimental condition of excitation power of $5.3 \times 10^{5} \mathrm{~W} / \mathrm{m}^{2}$, we estimate that the minimum number of $\mathrm{CdS}$ nanorods to have a detectable PL signal is approximately 100.

When the applied electric field is of $10 \mathrm{~V} / \mu \mathrm{m}$, it is found that the anisotropy of micro-PL spectrum drastically reduces, as shown in Figure 7. The reduced anisotropy is due to the reorientation of $\mathrm{CdS}$ nanorods along the $z$-axis driven by $\mathrm{LC}$ molecules. As described above, because the anchoring force

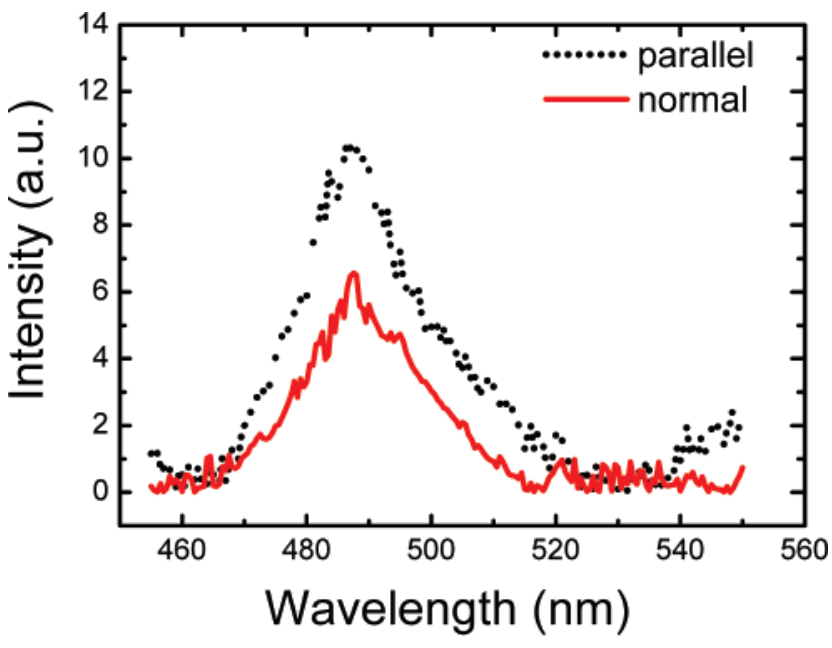

Figure 7. Microphotoluminescence spectra with the analyzer parallel (dashed line) and perpendicular (solid line) to the rubbed PI direction under an external electric field of CdS nanorods and LCs composite.

between nanorods and LCs can provide a large alignment energy, ${ }^{19}$ it enables CdS nanorods to reorient with LCs. As applying an external bias, LCs will be aligned along the direction of external electric field, and so do CdS nanorods, as shown in Figure 2b. Consequently, the polarization for the emission along the direction of external electric field is greatly reduced. According to previous reports, LCs will align along the direction of the external electric field in 20$30 \mathrm{~ms} .{ }^{26} \mathrm{We}$ therefore expect that the reorientation of the $\mathrm{CdS}$ nanorods should be in the same time range. When the electric field is turned off, the orientation of LC molecules will return to the rubbed PI direction also in the range of $20-30 \mathrm{~ms},{ }^{26}$ and so do the CdS nanorods. 


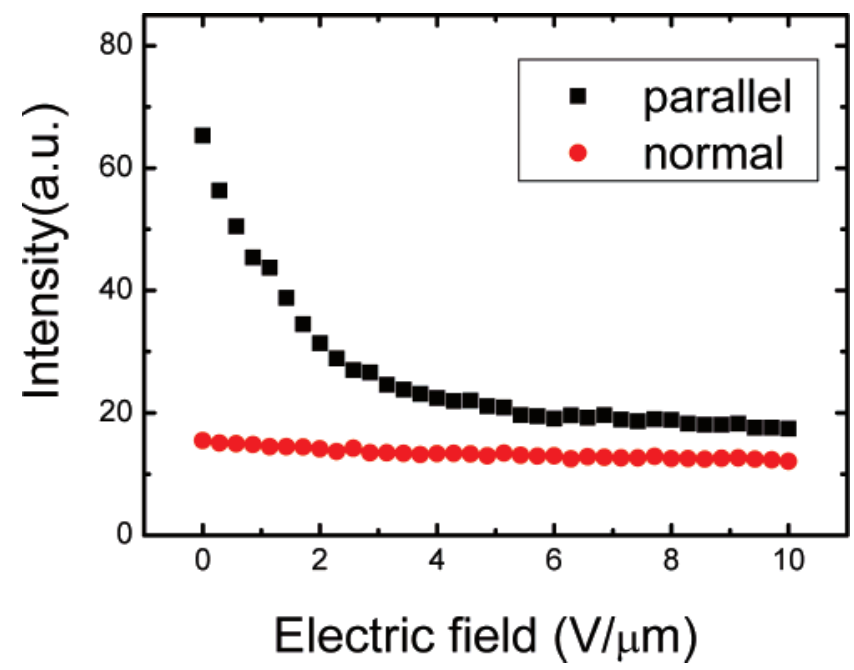

Figure 8. Intensity of microphotoluminescence vs external electric field with the analyzer parallel (solid square) and normal (circle) to the rubbed PI direction.

To examine the relationship between the polarization and external electric field, the intensities of micro-PL spectra with the analyzer parallel and perpendicular to the rubbed PI direction were measured as a function of external electric field, and the results are shown in Figure 8. The intensity with the analyzer parallel to the rubbed PI direction obviously decreases with external electric field and saturates at about $3 \mathrm{~V} / \mu \mathrm{m}$. In contrast, the intensity of micro-PL with the analyzer perpendicular to the rubbed PI direction is almost constant independent of the change of external electric field. The saturation of the anisotropy at about $3 \mathrm{~V} / \mu \mathrm{m}$ is consistent with the magnitude of the electric field to drive LC molecules from parallel to perpendicular orientation with respect to the rubbed PI direction. It thus provides an additional evidence to support our above proposal that the change of the observed anisotropy is driven by the reorientation of LC molecules.

Finally, to further stress that our observed optical anisotropy is indeed due to the reorientation of CdS nanorods driven by LC molecules, we have replaced LC molecules by toluene in the LC cells and found that the optical anisotropy vanishes, as shown in Figure 9. Similarly, if the LC molecules were replaced by water, the optical anisotropy also disappears. It implies that, without the assistance of LC molecules, CdS nanorods are randomly distributed, and thus the polarization of the emitted light from an ensemble of CdS nanorods disappears. We have therefore firmly established that our newly designed device based on the composite of LC and CdS nanorods possesses the unique property that the light emission arising from semiconductor nanorods can be electrically controlled. This behavior underpins the basic requirement for the future application in smart nanophotonic devices.

In summary, we have reported a new and general approach to manipulate the polarization of the emission from semiconductor nanorods by an external bias. Our novel device consists of a composite of liquid crystals and semiconductor nanorods. The unique property that the emission anisotropy

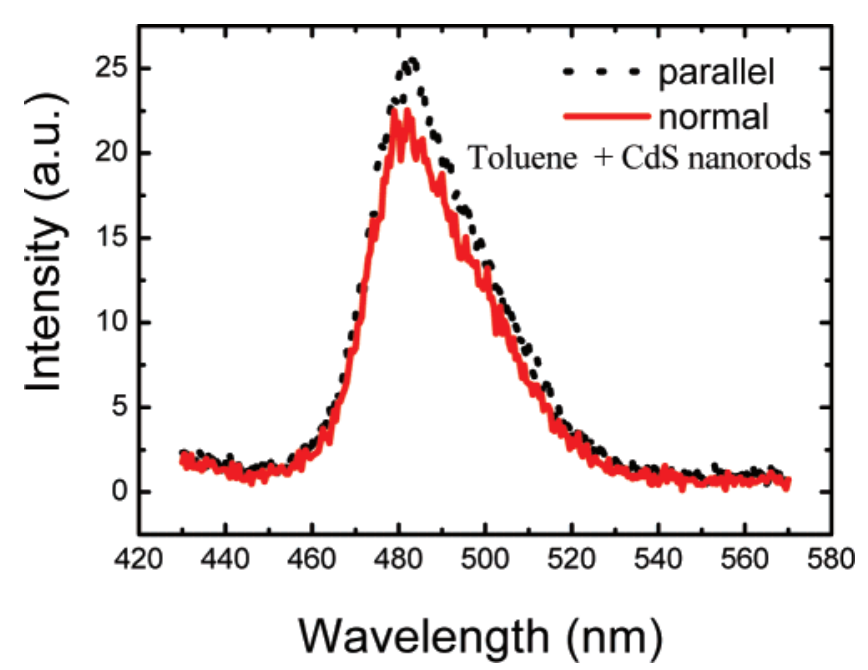

Figure 9. Microphotoluminescence spectra with the analyzer parallel (dashed line) and perpendicular (solid line) to the rubbed PI direction without an external electric field of $\mathrm{CdS}$ nanorods and toluene composite.

can be fine-tuned at will paves a new pathway for the application of one-dimensional semiconductor nanostructures in, e.g., optical switches, integrated photonic devices, as well as electrochromatic gadgets. Moreover, our approach, which utilizes the currently mature LCD technology, demonstrates an excellent advantage for the practical application in the near future.

Acknowledgment. This work was supported by the National Science Council of the Republic of China.

\section{References}

(1) Wang Z. L. Nanowires and Nanobelts: Material, Properties, and Devices; Kluwer Academic Publishers: Boston, 2003.

(2) Li, Y.; Qian, F.; Xiang, J.; Lieber, C. M. Mater. Today 2006, 9 (10), $18-27$.

(3) Pauzauskie, P. J.; Yang, P. D. Mater. Today 2006, 9 (10), 36-44.

(4) Lin, J. M.; Lin, H. Y.; Cheng, C. L.; Chen, Y. F. Opt. Lett. 2006 , $31,3173-3175$.

(5) Hu, J. T.; Li, L. S.; Yang, W. D.; Manna, L.; Wang, L. W.; Alivisatos, A. P. Science 2001, 292, 2060-2063.

(6) Lynch, M. D.; Patrick, D. L. Nano Lett. 2002, 2, 1197-1201.

(7) de Gennes, P. G.; Prost, J. The Physics of Liquid Crystals; Clarendon: Oxford, 1993.

(8) Gur, I.; Fromer, N. A.; Geier, M. L.; Alivisatos, A. P. Science 2005, $310,462-465$.

(9) Chen, C. Y.; Cheng, C. T.; Lai, C. W.; Hu, Y. H.; Chou, P. T.; Chou, Y. H.; Chiu, H. T. Small 2005, 12, 1215-1220.

(10) Poulin, P.; Cabuil, V.; Weitz, D. A. Phys. Rev. Lett. 1997, 79, 48624865.

(11) Poulin, P.; Stark, H.; Lubensky, T. C.; Weitz, D. A. Science 1997, $275,1770-1773$

(12) Loudet, J. C.; Barois, P.; Poulin, P. Nature 2000, 407, 611613.

(13) Andrienko, D.; Allen, M. P.; Skacej, G.; Zumer, S. Phys. Rev. E 2002, 65, 041702 .

(14) Lapointe, C.; Hultgren, A.; Silevitch, D. M.; Felton, E. J.; Reich, D. H.; Leheny, R. L. Science 2004, 303, 652-655.

(15) Lapointe, C.; Cappallo, N.; Reich, D. H.; Leheny, R. L. J. Appl. Phys. 2005, 97, 10Q304.

(16) Chu, K. C.; Chao, C. Y.; Chen, Y. F.; Wu, Y. C.; Chen, C. C. Appl. Phys. Lett. 2006, 89, 103107.

(17) Nelson, D. R. Nano Lett. 2002, 2, 1125-1129.

(18) Park, S. Y.; Stroud, D. Phys. Rev. Lett. 2005, 94, 217401.

(19) Burylov, S. V.; Raikher, Y. L. Phys. Rev. E 1994, 50, 358367. 
(20) Vouilloz, F.; Oberli, D. Y.; Dupertuis, M.-A.; Gustafsson, A.; Reinhardt, F.; Kapon, E. Phys. Rev. B 1998, 57, 1237812387.

(21) Venugopal, P.; Lin, P. I.; Liu, C. C.; Chen, Y. T. J. Am. Chem. Soc. 2005, 127, 11262-11268.

(22) Wang, J. F.; Gudiksen, M. S.; Duan, X. F.; Cui, Y.; Lieber, C. M. Science 2001, 293, 1455-1457.

(23) Qi, Jifa; Belcher, A. M.; White, J. M. Appl. Phys. Lett. 2003, 82, $2616-2618$.
(24) Akiyama, H.; Someya, T.; Sakaki, H. Phys. Rev. B 1996, 53, R4229R4232.

(25) Landau, L. D.; Lifshitz, E. M.; Pitaevskii, L. P. Electrodynamics of Continuous Media; Butterworth-Heinemann: Oxford, U.K., 1984; p 34.

(26) Pochi, Y.; Gu, C. Optics of Liquid Crystal Displays; Wiley: New York, 1999.

NL070541N 